\title{
Helioseismology in the 1980s and 1990s
}

\author{
Philip R. Goode
}

Big Bear Solar Observatory, New Jersey Institute of Technology

40386 N. Shore Lane, Big Bear City, CA, USA

email: pgoode@bbso.njit.edu

\begin{abstract}
Over more than twenty years, Wojtek Dziembowski and I collaborated on nearly fifty papers, which were concentrated in helioseismology through the 1980s and 1990s, but extended early into the new century. In this review, I discuss the most significant results of this collaboration and some of the underlying sociology that contributed to the intensity and longevity of our collaboration. Our work began with placing limits on the Sun's buried magnetic field and ended with extracting from the solar-cycle dependent oscillation frequency changes the roles (and net result) of competing dynamical drivers of changes in the solar diameter.
\end{abstract}

Keywords. Sun: helioseismology, Sun: oscillations, Sun: interior, Sun: photosphere

\section{Introduction}

In the 1970s, my research was concentrated on the problem of renormalizing the nucleon-nucleon interaction in the presence of a core nucleus. The problem was difficult because the renormalization of the two-nucleon interaction presented a poorly convergent perturbative expansion (see Goode \& Koltun 1975). The field was mature and progress was slow, which made the field one of diminished excitement. Then I learned of helioseismology and thought my experience in treating perturbations around complex spherical geometries would be applicable. However, my knowledge of astronomy was limited. Fortunately, Henry Hill introduced me to Wojtek Dziembowski and this began a collaboration that spanned more than twenty years. Wojtek's pioneering paper on nonradial oscillations had already become the handbook for the study of stellar oscillations (Dziembowski 1977) and I have worn out several copies.

Our collaboration began in earnest in 1982 and ended in 2005. It was my most productive, significant, educational and rewarding collaboration. In our collaboration, I typically spent one month each summer in Warsaw where we worked seated at desks facing each other. Then, Wojtek spent one month each winter in New Jersey where we shared an office and meals. I always looked forward to our time together because we would do something worthwhile scientifically, as we had fun and discussed all manner of topics. According to the paper count by A.A. Pamyatnych, our collaboration was Wojtek's most productive. It ended with a sense of fulfillment and sadness as we moved in different directions with his interest returning to the stars, and the wisdom of his choice is reflected in the astounding results that one sees in the proceedings of this conference and the central role of Wojtek in the field of asteroseismology. His work has been pioneering.

\section{The 1980s}

In 1983, we wrote a little known paper for the proceedings of the European Physical Society Meeting on Solar Oscillations held in Catania, Italy. The paper developed simple formulae describing, asymptotically, the effect of rotation and magnetic fields on solar oscillation frequencies. I still use the formulae in this paper (Dziembowski \& Goode 1984) 
and recommend it to people just learning helioseismology. Figure 1 is a picture of the two of us at that meeting. The picture was taken in the ruins of an ancient Sicilian amphitheatre and shows Wojtek making a scientific argument while I listen, think and learn more astronomy.

This was a truly exciting time in helioseismology. Duvall and Harvey (1984) had measured fine structure in the spectrum of the Sun's five-minute period oscillations from which we (Duvall et al. 1984) were able to determine the internal rotation of the Sun in its equatorial plane. We learned that the Sun has surface-like rotation down to its core in the equatorial plane, and so the Sun had "spun-down", which was somewhat of a surprise at the time. Shortly thereafter, Duvall et al. (1986) went to the South Pole and made more complete measurements of the fine structure in the oscillation spectrum from which they determined that surface-like differential rotation persisted beneath the surface. In two separate works in 1989, we determined that the surface-like differential rotation persisted to the base of the convection zone where there was a sharp transition to solid-body rotation (Brown et al. 1989, Dziembowski et al. 1989) at a mean surface rate. These papers used splitting data from Sacramento Peak Observatory (Brown \& Morrow 1987) and from Big Bear Solar Observatory (Libbrecht 1989).

\section{The 1990s}

In the early 1990s, we did our most mathematically pure work on stellar oscillations in which we developed a complete formalism, valid through second order in differential rotation $(\Omega(r, \theta))$, describing the effect of rotation on stellar oscillation frequencies (Dziembowski \& Goode 1992). We found that second-order effects on solar oscillation frequencies are dominated by effects of distortion, which must be accounted for in any effort to determine the Sun's internal magnetic field. For solar oscillations, accidental degeneracies can occur, but we found that they cannot lead to large frequency shifts. However for evolved $\delta$ Scuti stars, calculated spectra are dense, and due to rotational

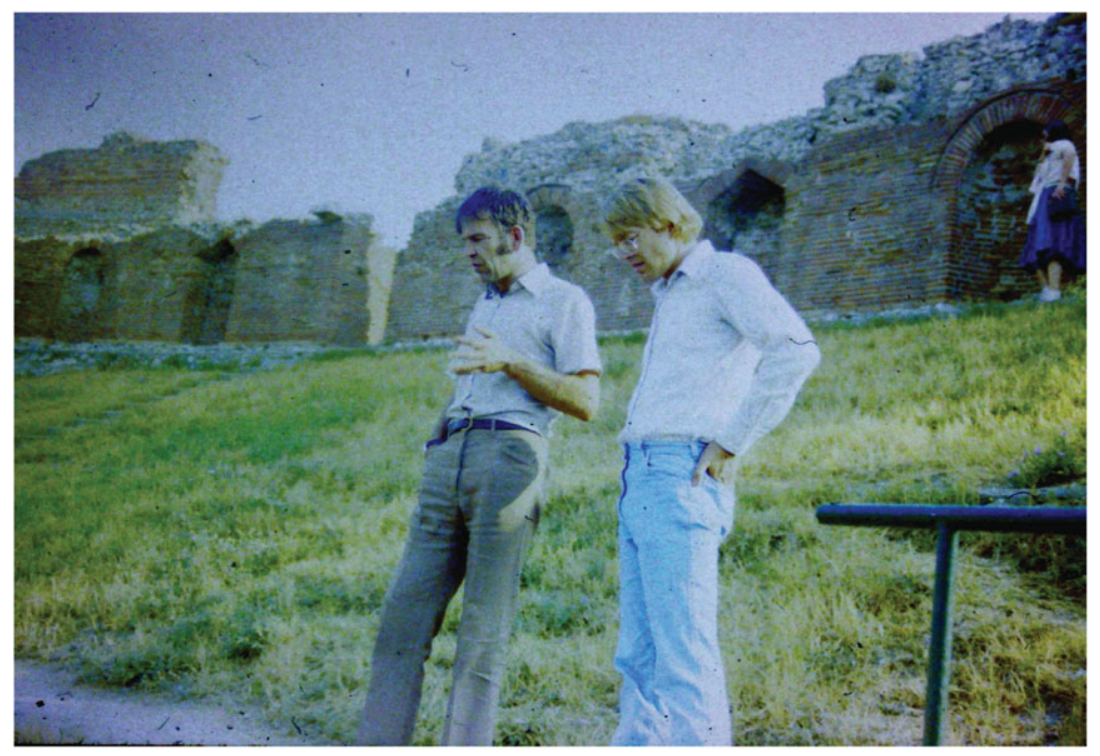

Figure 1. Dziembowski and Goode in Catania, 1983. 
perturbation members of neighboring multiplets may overlap. Here, we emphasized the seismic potential of modes with mixed p- and g-mode character. The paper was frustrating in that a large effort did not lead to a hoped-for significant second-order effect of rotation on solar oscillations, but was satisfying in its completeness.

The problem of the Sun's neutrino deficit had been the subject of endless controversy beginning with the magnificent work of Ray Davis that ultimately led to his Nobel Prize. After Davis's work was accepted, the question was whether or not there was an astrophysical solution to the Sun's neutrino paradox (aside: with typical incisive humor Wojtek described this as determining who owns the universe - astrophysicists or particle physicists). We inverted p-mode frequencies from Libbrecht's BBSO data, plus BiSON data for the low- $l$ modes, to obtain the run of pressure, density and mass, as well as the fractional surface helium abundance - the seismic solar model (Dziembowski et al. 1994, 1995). We assumed that gravity and pressure are the only forces acting and that the adiabatic exponent is close to the model value except in the outmost layers. To obtain the run of the temperature, further assumptions would have been required. The results of the seismic model agreed quite well with the standard model. Several variations of the standard model used were considered: a young Sun, p+p enhanced by 1.034 (this reaction occurs at too low an energy to be measured in the laboratory, and so there is some uncertainty in it), the ${ }^{3} \mathrm{He}+{ }^{3} \mathrm{He}$ part of the p-p chain enhanced by a factor of nine (under the unlikely assumption of a low-energy resonance in the reaction, which has the effect of circumventing the requisite two-thirds of the p-p chain that produces neutrinos with sufficient energy to be detected in the Davis's deep underground Homestake Mine experiment in which ${ }^{37} \mathrm{Cl}$ is converted to ${ }^{37} \mathrm{Ar}$ in a gigantic tank of cleaning fluid). The deviations from the standard with seismic models determined with the variations led to several conclusions. First, there is no astrophysical solution to the solar neutrino problem (the Sun is prosaic and the particle physicists "own the universe"). Second, the seismic age of the Sun is comparable to that determined from meteorite data.

An aside: During this same time period, we attended a helioseismology meeting at the Tata Institute set to coincide with a total eclipse of the Sun in 1995. During this time, we hired a car and took a two-man (plus driver) tour for several days to many sites of interest. Among other adventures, we found ourselves in a line for a crematorium on the banks of the Ganges in the holy city of Varanasi, and in another city were chased from a hotel room by a "giant" white lizard. The highlight of the trip was the total eclipse where we selected the playing field of a boys school as our place to view it. While I was setting up to watch, I turned and saw Wojtek surrounded by teenage schoolboys listening to him giving an extemporaneous talk about the Sun and solar eclipses. A great, but also typical moment. On that trip, as usual, all topics were open for discussion, and often discussed. We also had a daylong adventure to visit the Taj Mahal (see Fig. 2).

Later in the 1990s, we used MDI/SoHO data on f mode frequencies to determine the seismic radius of the Sun. We found that Kosovichev and Schou had the same idea, so we published the result in a single paper (Schou et al. 1998). The principle was simple: we noted that high- $l$ f modes (in this case $l=88-250$ ) are surface waves described by a simple formula for the angular frequency in which $\omega \approx \sqrt{G M_{\odot} / R_{\odot}{ }^{3}}$, where $G$ is the universal gravitational constant and $M_{\odot}$ is the mass of the Sun, while $R_{\odot}$ is photospheric radius of the Sun. With this we determined a value of the photospheric radius of the Sun that was $0.3 \mathrm{Mm}$ smaller than the textbook value (Allen 1973). Christensen-Dalsgaard \& Brown (1998) subsequently showed that this difference was due to a $0.3 \mathrm{Mm}$ difference between the true photospheric radius and that measured by transit. Thus, the radius in standard solar models had to be reduced by $0.3 \mathrm{Mm}$. 


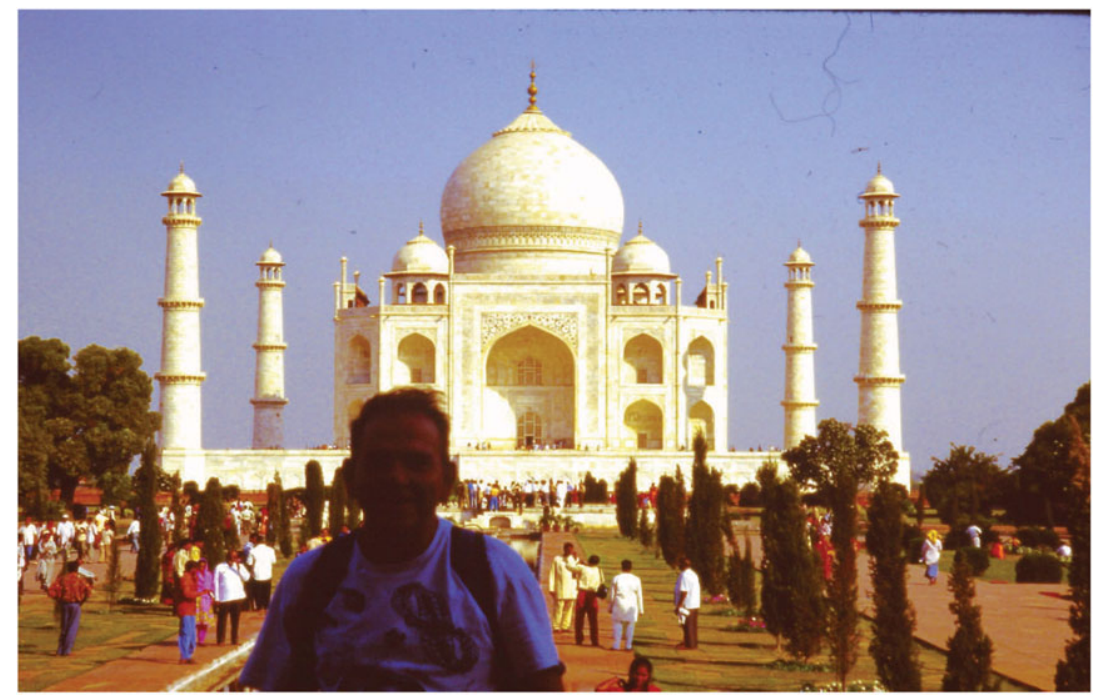

Figure 2. 1995 at the Taj Mahal.

\section{Ending the collaboration}

Early in the new century, after twenty years of close collaboration, global helioseismology had become a mature science and neither of us had an interest in switching to local helioseismology. With Kepler and CoRoT coming, Wojtek would return to his first love and the wisdom of that choice is written all over this conference. As for me, I wanted to build a solar telescope. So we agreed on one last piece of work to address a number of problems that had vexed us over the years and end the collaboration on a high note.

It had long been known that both $\mathrm{f}$ - and $\mathrm{p}$-mode frequencies (after removing mode inertia) closely track the solar cycle and increase with increasing solar activity. However, they do not show the same frequency dependence. In particular, f-mode frequencies abate with increasing mode frequency, while p-mode frequencies increase more strongly with increasing frequency. From this, we (Dziembowski \& Goode 2005) were able to demonstrate, in a nearly self-consistent manner, that both do not have the same origin. The f-mode behavior is due to rising field effects primarily about $5 \mathrm{Mm}$ beneath the surface, while the field growth is much smaller near the surface as observed (Lin 1995, Lin \& Rimmele 1999), which is the region to which higher frequency $\mathrm{f}$ modes are more sensitive. Such field growth near the surface is too weak to directly affect the p-mode frequencies. Rather, the field growth very slightly blocks the turbulent pressure and the heat flow and the resulting cooling shrinks the outer cavity to net the measured p-mode behavior. The final result is a Sun that shrinks $\sim 1 \mathrm{~km}$ from activity minimum to activity maximum, far too small to be of climatological significance and further presents no reason to imagine significant changes in the solar diameter over historical timescales.

\section{Afterwards}

Wojtek has enjoyed a time of great interest in the field of his first love since our collaboration ended. I changed fields to build a new solar telescope (NST).

The NST is the first facility-class telescope built in the US in a generation. Its $1.6 \mathrm{~m}$ aperture was chosen because it is the smallest aperture that can resolve features of order 


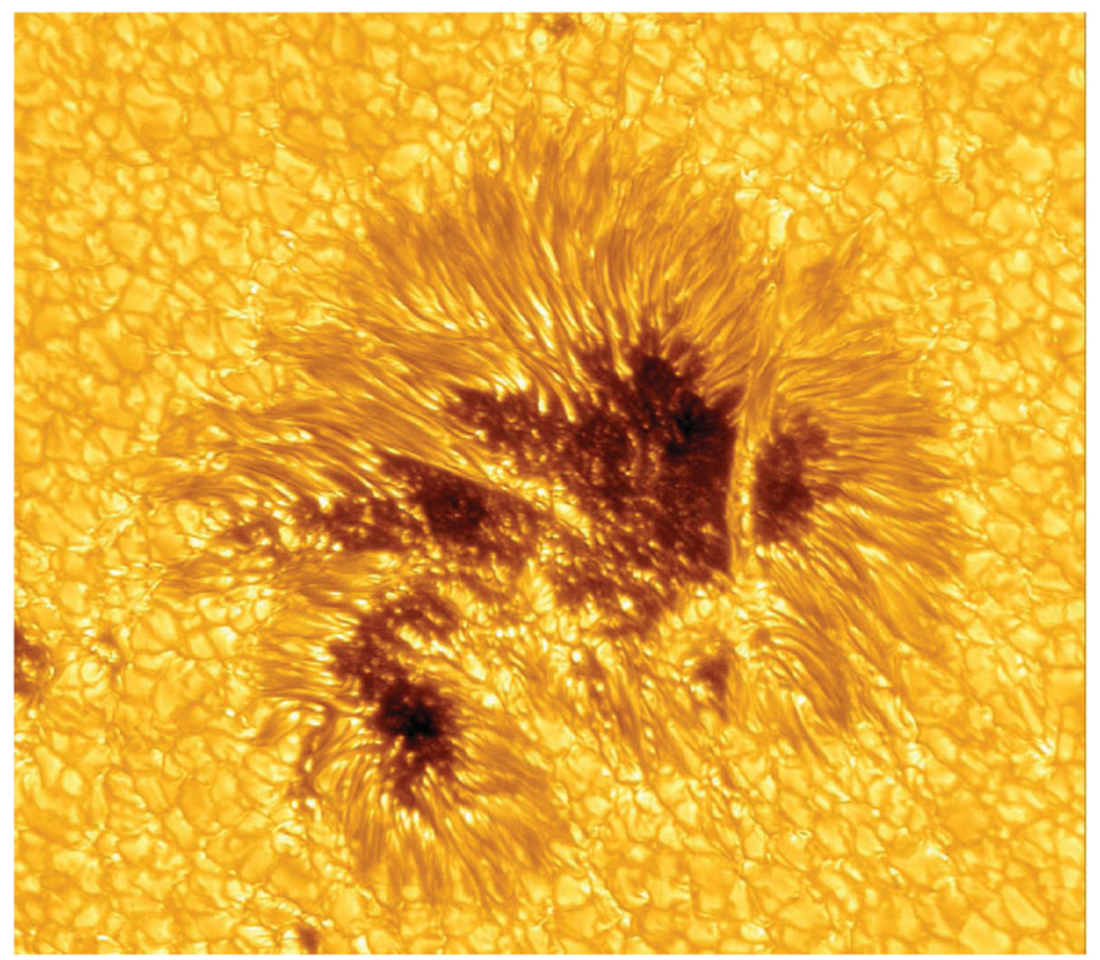

Figure 3. NST sunspot from 22 May 2013. These observations are able to resolve a darkness at the center of umbral dots and fine structure in the light bridge.

$100 \mathrm{~km}$ (diffraction limit of $\sim 40 \mathrm{~km}$ ). This matters because the photon mean free path in the solar photosphere is $\sim 100 \mathrm{~km}$. After several years of effort, the telescope saw first light in January 2009 and with improving instruments and new generation adaptive optics (AO), the telescope now reaches its diffraction limit in the bluest visible wavelengths. Observations with AO regularly are made with lock of several hours. An image of a sunspot is shown in Fig. 3.

\section{Acknowledgements}

P.R.G gratefully acknowledges partial support by NASA (NNX13AG14G), NSF (AGS1250818 and AFOSR (FA2386-12-1-3018).

\section{References}

Allen, C. W. 1973, in Astrophysical Quantities, 3rd Ed., p. 169

Brown, T. M., Christensen-Dalsgaard, J., Dziembowski, W. A., Goode, P. R., Gough, D. O., \& Morrow, C. A. $1989, A p J, 343,526$

Brown, T. M. \& Morrow, C. A. 1987, ApJ, 314, L21

Christensen-Dalsgaard, J. \& Brown, T. M. 1998, ApJ, 500, 195

Duvall, T. L., Dziembowski, W. A., Goode, P. R., Gough, D. O., Harvey, J. W., \& Leibacher, J. W. 1984, Nature, 310, 22

Duvall, T. L. \& Harvey, J. W. 1984, Nature, 310, 19

Duvall, T. L., Harvey, J. W., \& Pomerantz, M. 1986, Nature, 321, 500

Dziembowski, W. A. 1977, AcA, 27, 203

Dziembowski, W. A. \& Goode, P. R. 1984, MemSAI, 55, 185

Dziembowski, W. A. \& Goode, P. R. 1992, ApJ, 394, 670 
Dziembowski, W. A. \& Goode, P. R. 2005, ApJ, 625, 548

Dziembowski, W. A., Goode, P. R., \& Libbrecht, K. G. 1989, ApJ, 337, L53

Dziembowski, W. A., Goode, P. R., Pamyatnykh, A. A., \& Sienkiewicz, R. 1994, ApJ, 432, 417

Dziembowski, W. A., Goode, P. R., Pamyatnykh, A. A., \& Sienkiewicz, R. 1995, ApJ, 445, 509

Goode, P. R. \& Koltun, D. S. 1975, Nucl. Phys. A, 243, 44

Libbrecht, K. G. 1989, ApJ, 336, 1092

Lin, H. 1995, ApJ, 514, 448

Lin, H. \& Rimmele, T. R. 1999, ApJ, 446, 421

Schou, J., Kosovichev, A. G., Goode, P. R., \& Dziembowski, W. A. 1997, ApJ, 489, L197 